\title{
Agendas de género en las campañas presidenciales de 2018 en México*
}

\section{Gender Agendas in the 2018 Presidential Campaigns in Mexico}

\author{
GILBERTO MORALES** \\ ESPERANZA PALMA***
}

\begin{abstract}
This article analyzes gender related issues posed by three presidential candidates nominated by partisan coalitions in the 2018 elections. The coalition's electoral platforms were reviewed and, drawing from journalistic information, a data base was constructed in which all of Lopez Obrador's, Meade's and Anaya's events during their campaigns were registered. The main conclusion is that, in their agendas, the topics of gender from a distributive approach that reinforces traditional roles for women are predominant. In a parallel way, various feminist and LGBT+ collectives presented agendas for equality, which have been as a consequence represented by alternative communities.
\end{abstract}

Key words: political agenda, gender, elections, alternative communities

\begin{abstract}
Resumen
Este artículo se propone analizar los temas relativos al género abordados por los tres candidatos a la Presidencia postulados por las coaliciones partidistas en las elecciones de 2018. Se examinaron las plataformas electorales de las coaliciones $y$, a partir de información periodística, se construyó una base de datos en la cual se registraron todos los eventos de López Obrador, Meade y Anaya durante las campañas. La conclusión central es que, en las agendas, predominaron los temas de género de corte distributivo que refuerzan los roles tradicionales de las mujeres. De manera paralela, varios colectivos feministas y LGBT+ colocaron agendas por la igualdad, constituyéndose así en públicos alternativos. Palabras clave: agenda política, género, elecciones, públicos alternos
\end{abstract}

$\mathrm{L}$ as campañas electorales representan una oportunidad para analizar las posiciones de los actores partidistas en torno a algunos temas públicos. Quienes compiten por el poder político, en sus distintos niveles, transmiten, cara a cara con el electorado, la plataforma sobre la que se cimentará el ejercicio de dicho poder. En México, durante el tiempo de las campañas presidenciales, quienes contienden tratan de convencer a la ciudadanía de que su propuesta política es la mejor opción.

La estrategia de campaña debe considerar, entre otras variables, el diseño de una agenda política cuyos contenidos aborden y brinden "soluciones" a los temas de interés público, que deben atenderse con urgencia

* Artículo recibido el 13/11/18 y aceptado el 13/02/19.

** Universidad Autónoma Metropolitana, Unidad Azcapotzalco, Departamento de Sociología. Av. San Pablo Xalpa núm. 180, col. Reynosa Tamaulipas, Azcapotzalco, 02200, Ciudad de México <gilcamus@gmail.com>. orciD: https: / /orcid.org/O0000001-6450-5663.

*** Universidad Autónoma Metropolitana, Unidad Azcapotzalco, Departamento de Sociología. Av. San Pablo Xalpa núm. 180, col. Reynosa Tamaulipas, Azcapotzalco, 02200, Ciudad de México <eipc2000@yahoo.com>. 
$\mathrm{y}$ que se interpretan como asuntos relevantes para la población. Las agendas siempre abarcan temas diversos relativos al ámbito económico, laboral y de seguridad, a la educación, la salud, el desarrollo social y urbano y la cultura. De manera secundaria, las agendas electorales incluyen un análisis de la situación social de las mujeres (empleo, salario, aborto, violencia de género, paridad y desarrollo político), y de las personas de la diversidad sexual (matrimonio igualitario, adopción homoparental, derechos civiles y políticos). Si bien estas cuestiones están presentes en las plataformas de los partidos, en las campañas son tópicos secundarios (Sapiro y Johnston, 1997).

Las elecciones presidenciales de 2018 en México no fueron la excepción. Ninguno de los candidatos a la Presidencia se comprometió con una agenda de género, aunque aisladamente abordaron temas relacionados con las mujeres. Lo anterior plantea un problema, dado que en México el número de mujeres en los cargos de representación ha aumentado de modo considerable en los últimos años, gracias a las cuotas de género y a la paridad como parte de un proyecto de democracia paritaria (Cobo, 2004), al grado de que en la actualidad el Congreso y el gabinete son prácticamente paritarios. ¿Cómo se explica esta paradoja de que la mayor presencia de mujeres en política, tanto como candidatas como en los cargos de representación, no se corresponde con agendas sustantivas de género en las campañas? ¿Cuál sería, en todo caso, una agenda por la igualdad de género?

A partir de estas preguntas, el presente ensayo se propone dos objetivos: a) analizar el subtexto de género en las agendas y plataformas electorales presentadas en las campañas presidenciales de 2018 por los tres candidatos más importantes: Andrés Manuel López Obrador (AMLO), José Antonio Meade y Ricardo Anaya; b) hacer un mapeo de los públicos alternos que surgieron durante la campaña, esto es, los colectivos feministas y LGBT+, que en paralelo a las campañas generaron sus propias agendas y pidieron a los candidatos que se comprometieran con éstas.

La metodología consistió en analizar las plataformas electorales de los partidos que conformaron las coaliciones de los tres contendientes a la Presidencia, así como los temas referentes al género que trataron durante las campañas. Para ello, se construyó una base de datos novedosa donde se registraron todos los eventos y los temas que tocó cada candidato del 30 de marzo (inicio de las campañas) al 10 de junio (término de las mismas); las fuentes utilizadas fueron periódicos nacionales y locales: El Financiero, El Sol de México y sus versiones locales (El Sol de Tijuana, El Sol de Puebla, etcétera), El Universal, Excélsior, La Jornada y sus versiones locales (La Jornada Aguascalientes, La Jornada Maya, etcétera), Milenio, Proceso, así como los sitios de Facebook y Twitter de los candidatos.

Este artículo comprende tres apartados. El primero recoge un debate sobre la teoría de la masa crítica y algunos planteamientos sobre la construcción del concepto de género en política; el segundo analiza las propuestas de cada candidato a lo largo de la campaña, así como sus plataformas electorales; el tercero examina los públicos alternativos: los colectivos feministas y LGBT+, cuya exigencia fue que sus agendas fueran tomadas en cuenta. Como se mostrará, los candidatos se vieron obligados a discutir lo que puede llamarse una agenda de género, sobre todo en sus campañas. Finalmente, en las conclusiones se reflexionará en torno a una posible respuesta a la paradoja enunciada arriba. Si la presencia, cada vez más acentuada y visible de las mujeres en los espacios políticos no se traduce en una agenda de género en las campañas, eso se explica en parte por cómo se comprende políticamente el género. En otras palabras, se sostendrá que las agendas de género en campañas soslayan conceptos normativos, como el de libertad, en aras de una visión esencialista de los sujetos-electores (colectivos e individuales), lo que responde, no obstante, a ciertas necesidades de carácter identitario, así como a certezas ideológicas y ontológicas, lo cual resulta contradictorio, pues la rentabilidad política puede estar basada en divisiones y confrontaciones políticas y sociales que no desean los candidatos contendientes.

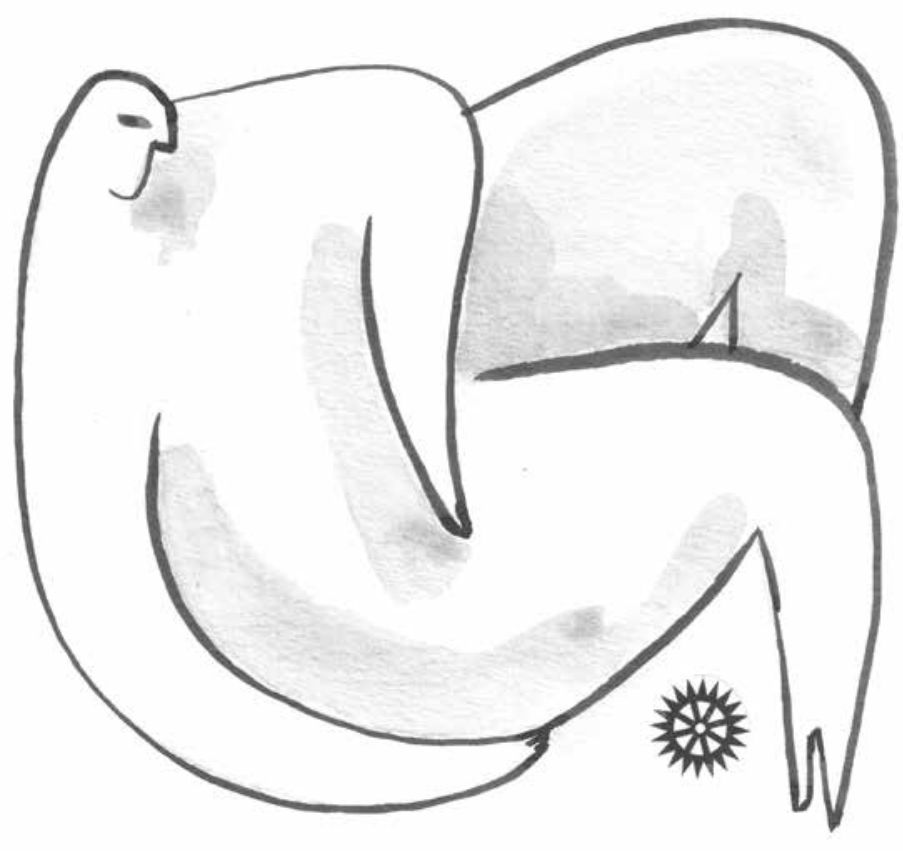




\section{El debate académico}

Las preguntas elaboradas en este ensayo derivan de algunos debates de los estudios de género y la representación y de la teoría feminista.

La incongruencia que supone, por un lado, un mayor número de mujeres en las candidaturas y en los cargos públicos $\mathrm{y}$, por otro, la irrelevancia de los temas de género en las campañas se presenta desde la teoría de la masa crítica. De acuerdo con esta teoría, el incremento del número de mujeres en la política, en particular en el legislativo, permite que éstas sean más visibles, que trabajen juntas de manera consistente en políticas con perspectiva de género, y eso hará más probable que influyan a sus colegas hombres para conseguir apoyo político (Kanter, 1977). Según esta tesis, la representación descriptiva (esto es, la creciente presencia de mujeres en el ámbito legislativo) tendría un impacto en la representación sustantiva, en las agendas y en las políticas públicas. Es pertinente plantear que una mayor presencia de candidatas, como resultado de la paridad, abriría la posibilidad de colocar temas de género en las campañas y en el debate público. Empero, dicha tesis no se confirma en este estudio ni en otros que han analizado el creciente número de mujeres en los parlamentos (Studlar y McAllister, 2002; Childs y Krook, 2006). Pese a la mayor cantidad de candidatas y de mujeres congresistas en las campañas de 2018 no se ofrecieron agendas por la igualdad que posicionaran temas cruciales como el aborto o la brecha de género en diversos ámbitos de la vida social y laboral. La definición de una agenda de género está determinada por distintas percepciones de lo que es el género, por un posicionamiento ideológico y por cálculos electorales que tendrán en cuenta a un electorado de base y uno personal (Reyes-Housholder, 2018) que no necesariamente se preocupa por temas de esa naturaleza.

Así, una de las cuestiones centrales es ¿cómo entienden las y los políticos la agenda de género? El análisis de los temas y el tratamiento político relativos a las "mujeres" durante la campaña revelan cuestiones de carácter identitario, que parten de una óptica del orden social y del lugar que deben ocupar las mujeres en dicho orden. Lo interesante es que, como describiremos, los candidatos se vieron obligados a discutir y a incluir parcialmente en sus agendas temas asociados con la igualdad de género $\mathrm{y}$, desde un criterio más amplio, con la libertad, ante las presiones de contrapúblicos alternos, esto es, con terrenos discursivos paralelos en donde los colectivos subordinados se inventan y circulan contradiscursos (Fraser, 1993). Lo anterior indica que la movilización de actores con agendas por la igualdad son centrales para que los temas de género sean visibilizados durante las campañas. Y esto es justo lo que apreciamos en las campañas: por un lado, los discursos de los candidatos $\mathrm{y}$, por otro, los de actores alternos con agendas feministas.

El tratamiento político que se da a las mujeres en las campañas toca un problema abierto por la teoría feminista: ¿cómo deben ser interpeladas las mujeres políticamente, como individuos o como mujeres? (Serret, 2016). Las propuestas de los candidatos, como se advertirá, responden a:

La homogeneización de las personas en virtud de su género que emprende esta lógica que solo puede explicarse por la ilusión imaginaria que encuentra tranquilidad en las certezas de un mundo dividido en estratos, en el que las claras diferencias entre grupos (que no entre individuos) ayudan a saber, con solo mirarlas, cómo debemos clasificar a una persona, qué podemos esperar de ella y, en consecuencia, cómo debemos actuar nosotras y nosotros mismos [Serret, 2016: 22]

Al analizar los temas de género debe entenderse que las mujeres no son un grupo homogéneo y que ellas mismas y las élites políticas tienen distintas versiones de los temas que les atañen al ser tratadas en cuanto grupo y no en cuanto individuos. Así, es preciso distinguir entre agendas de corte distributivo-asistencial y las de corte igualitario.

Por ejemplo, en un análisis sobre la agenda de género en las campañas electorales para la Presidencia de la República del 2012, Cerva y Cornejo (2014: 7) señalan que una agenda política concatena una serie de procesos políticos y sociales que, en contextos democráticos, despliega un escenario donde distintos actores políticos negocian, debaten e intercambian propuestas sobre temas que se consideran importantes para ser atendidos políticamente. Los acuerdos cristalizan en promesas y propuestas electorales que trascenderán como políticas públicas. Una agenda, por lo tanto, no sólo tiene su origen a partir de la ideología de un partido, sino que es producto de debates, cabildeos, implícitos o explícitos, entre las fuerzas políticas institucionalizadas y sectores más amplios de la sociedad.

El estudio citado describe los temas concernientes a las mujeres presentados por Andrés Manuel López Obrador (Partido de la Revolución Democrática, PRD), Enrique Peña Nieto (Partido Revolucionario Institucional, PRI) y Josefina Vázquez Mota (Partido Acción Nacional, PAN) y la conclusión medular es que el género estuvo ausente de las plataformas electorales. Todas las agendas, unas más, otras menos, adolecen de una miopía conceptual, cuya consecuencia es que 
las propuestas tienden a esencializar los roles de las mujeres como amas de casa, madres, cuidadoras e integradoras del orden familiar. No se promueve, en consecuencia, una igualdad entre hombres y mujeres, es decir, una agenda que coloque en el centro el concepto de ciudadanía, sino que, en el mejor de los casos, se proponen alternativas para paliar asuntos privados de los que adolecen las mujeres (Cerva y Cornejo, 2014: 9).

Estos temas se vinculan a lo que Schaffner (2005) describe como cuestiones sobre mujeres. En una investigación donde analiza la propaganda (principalmente televisiva) de las campañas de 30 senadores en el año 2000 en Estados Unidos, el autor sostiene que, a partir de los años ochenta, el género ha tenido influencia en el comportamiento político estadounidense (entendiendo género como mujeres). Desde entonces, las mujeres tienden a votar por los demócratas porque sus preferencias políticas son más liberales (Schaffner, 2005: 803).

De tal suerte, en distintas campañas electorales, los demócratas estadounidenses han tratado de incrementar el número de mujeres votantes a su favor incluyendo temas de su particular interés. Éstos forman parte de políticas de bienestar social. Las mujeres estadounidenses, más que los varones, se han visto beneficiadas, por ejemplo, con programas sociales, en los cuales se incluyen cuestiones como la educación, el cuidado de las crías, la seguridad médica y social. Medidas asistenciales que inciden de forma directa en la vida de las mujeres, por ello se ven más inclinadas a votar por los demócratas que por los republicanos, quienes no comparten la visión ideológica de sus contrincantes. Lo interesante del planteamiento de Schaffner es que en ningún momento sostiene que dichas cuestiones remitan a un esencialismo $u$ ontologización de las mujeres. Es decir, las políticas o medidas asistencialistas responden a la inclinación ideológica demócrata, por lo que no se proponen como cuestiones de género en su agenda electoral. En todo caso, las mujeres se sienten identificadas con las medidas de bienestar social, por lo tanto, su voto se orienta hacia el partido demócrata. La lógica es inversa en el ámbito político-electoral mexicano: al final todas las coaliciones incluyeron temas de género, pero desde una mirada que afianzaba roles tradicionales. Como se mostrará, en 2018 encontramos la preeminencia de los contenidos asistenciales: apoyos económicos a las familias, apoyo a la niñez, creación de estancias y guarderías, horarios laborales flexibles para las madres trabajadoras, aunque también, de manera marginal, se abordaron otros asuntos como la igualación de los salarios entre hombres y mujeres, propuestas para garantizar el respeto de las mujeres en todos los espacios sociales, así como su presencia en los ámbitos políticos de toma de decisiones; el etiquetamiento de recursos económicos con perspectiva de género, el desarrollo y la consolidación de una cultura contra la violencia hacia las mujeres, que son parte de una agenda por la igualdad de género.

\section{Las agendas de género en las campañas presidenciales de 2018}

Como se mencionó, las agendas de género se reconstruirán mediante el análisis de las plataformas electorales de las principales fuerzas políticas del país (Movimiento de Regeneración Nacional [Morena], PRI y PAN) y de un seguimiento mediático de la campaña electoral de los candidatos. En el caso de las plataformas electorales, todos los partidos políticos, con excepción del Partido Verde Ecologista de México (PVEM), consideran la equidad de género como un objetivo importante. Con sus respectivos matices, postulan que este rubro es un eje sobre el que debe trabajarse con políticas públicas particulares, por lo tanto, ningún candidato pudo eludir los temas de género. Pero, como se expondrá, sus propuestas están ancladas en una visión esencialista de los sujetos.

\section{Andrés Manuel López Obrador}

La coalición Juntos Haremos Historia, conformada por Morena, el Partido del Trabajo (PT), ambos de izquierda, y por el conservador Partido Encuentro Social (PES), representó la opción más progresista en las elecciones de 2018.

En la plataforma electoral de Morena $^{1}$ la equidad de género se considera un aspecto fundamental del proyecto de nación, lo que significa garantizar los derechos de las mujeres a través de políticas públicas que atiendan, por ejemplo, las expresiones de violencia en contra de las mujeres. En el mismo documento, se admite que la igualdad entre hombres y mujeres está reconocida en la ley, aunque, en los hechos, ellas se ven sujetas a una serie de prácticas que violentan su seguridad y bienestar. De tal modo, sostiene el partido, su proyecto, en cuanto incluyente, debe eliminar

\footnotetext{
1 Morena, Proyecto alternativo de nación 2018-2024. Plataforma electoral y programa de gobierno <https://repositorio documental.ine.mx/xmlui/bitstream/handle/123456789/94946/CGor201801-31-ap-20-8-al.pdf> [6 de noviembre de 2018].
} 
todos los obstáculos que impiden el pleno desarrollo de las mujeres y su participación en los distintos aspectos de la vida social en igualdad de condiciones que los varones.

Una reflexión sobre la agenda de género en la campaña de Andrés Manuel López Obrador no puede soslayar la visión de los partidos coaligados con Morena sobre la cuestión de género. Ello por dos razones: primero, porque fue la coalición política victoriosa en las elecciones; segundo, porque el Partido Encuentro Social es abiertamente reaccionario, lo que, en términos ideológicos, lo coloca en el espectro político que se opone a los derechos de las mujeres en ciertos ámbitos, por ejemplo, en la libertad sobre sus cuerpos, y que rechaza las sexualidades diversas.

En la plataforma electoral del PT, dentro del rubro "Ámbito social", ${ }^{2}$ se precisa como cuarto punto el tema Mujeres y democracia. Se reconoce, de inicio, que la igualdad de género y los avances al respecto en nuestro país se deben a la lucha de las mujeres, es decir, propiamente al feminismo y a organizaciones civiles en México. Bajo esa tesitura, puede confirmarse el aspecto confluente entre distintas fuerzas y públicos de las agendas político-electorales, pues, como señala la plataforma del Pт, los temas referentes a las relaciones sociales, de pareja, familiares y del acceso a cargos públicos en condición de igualdad han sido llevados al terreno de la discusión pública por las mujeres. El PT propone una serie de medidas culturales, legislativas, de acción afirmativa y políticas públicas para erradicar la violencia de género en todas sus manifestaciones, garantizar el acceso de las mujeres sin discriminación a actividades laborales, la libre maternidad, la capacitación y derechos laborales.

Por su parte, en su plataforma electoral, el PES $^{3}$ argumenta que la transversalidad de la perspectiva de género es fundamental para lograr cambios estructurales en nuestro país y de este modo lograr una igualdad entre hombres y mujeres. De manera inmediata, se desarrolla la primera de cinco áreas que el PES identifica como de mayor importancia: "Vida y familia", dentro de la cual se exponen distintas propuestas para "proteger de manera transversal la vida". A partir de este punto se logra visibilizar la ideología conservadora del partido.
Más adelante se realizará un análisis de las implicaciones que las propuestas del pes tienen con respecto a la igualdad de las mujeres y la concepción de lo que debe ser una mujer, porque cristalizan a la perfección el imaginario de la identidad de género en nuestra sociedad. Por el momento, basta con enunciarlas: 1) se comprometen a proteger la vida desde la concepción como un derecho fundamental; 2) garantizar la protección a la familia y el reconocimiento del matrimonio como la unión entre un hombre y una mujer; 3) promover educación matrimonial como medida para solucionar conflictos familiares; 4) crear la Secretaría Nacional de la Familia; 5) promover espacios específicos para mujeres en proceso de lactancia en el sector público y privado.

Así se puede sintetizar el contenido electoral de Juntos Haremos Historia por lo que toca a las cuestiones sobre las mujeres y el género. Ahora bien, esa discursividad, ¿cómo se llevó a cabo en la campaña territorial?

Durante su campaña, Andrés Manuel López Obrador visitó todo el país y realizó un total de 209 eventos. Pero sólo en uno de ellos, según el seguimiento efectuado, aludió a la cuestión de género: en Apodaca, Nuevo León, ofreció becas para madres solteras que estudian una carrera universitaria. Sólo esa mención a lo concerniente al género. En efecto, como se sabe, su campaña se centró en la crítica a la corrupción, sacar del poder a la mafia y, con ello, generar la denominada Cuarta Transformación.

Los temas eje de las plataformas electorales de su partido, así como los de la coalición, en buena medida quedaron marginados, entre ellos, por supuesto, el de la igualdad entre hombres y mujeres. De hecho, cuando se le preguntaba abiertamente sobre el aborto y el matrimonio entre personas del mismo sexo, respondía más a la agenda del pes que a la de Morena o del PT: "son temas que la ciudadanía tiene que decidir... yo tengo una postura: eliminar la corrupción". ${ }^{4}$ Así lo manifestó en "Los diálogos con estudiantes de la Universidad Iberoamericana". ${ }^{5}$

Sin embargo, frente a los pronunciamientos de los colectivos feministas, sus asesoras Tatiana Clouthier y Olga Sánchez Cordero lanzaron la posición del Femsplaining con temas sobre educación, inclusión

2 Partido del Trabajo, Plataforma electoral 2018-2024<https://repositoriodocumental.ine.mx/xmlui/bitstream/handle/ 123456789/94967/CGor201801-31-ap-20-4-a1.pdf> [6 de noviembre de 2018].

3 Partido Encuentro Social, Plataforma electoral nacional. Proceso electoral 2017-2018 <https: / / repositoriodocumental.ine. $\mathrm{mx} / \mathrm{xmlui}$ /bitstream/handle/123456789/94970/CGor201801-31-ap-20-9-a1.pdf> [6 de noviembre de 2018].

4 Para una referencia puntual, véase la nota periodística: Cinco feministas opinan sobre el "Femsplaining", las propuesta de género de López Obrador <https: / /verne.elpais.com/verne/2018/06/02/mexico/1527892253_983452.html> [10 de noviembre de 2018].

5 Para la referencia exacta, consúltese Verificado 2018 <https://verificado.mx/aborto-matrimonio-igualitario-mariguanatemas-eleccion/> [10 de noviembre de 2018]. 
laboral, trabajo doméstico, feminización de la pobreza, violencia, acceso a la justicia y administración pública con perspectiva de género. No obstante, evadieron el tema del aborto para no entrar en franca contradicción con el candidato (Barragán y Rodríguez, 2018).

\section{José Antonio Meade}

La plataforma electoral del $\mathrm{PRI}^{6}$ para la contienda presidencial de 2018 estuvo articulada en torno a cinco ejes (economía abierta, seguridad pública, educación, sociedad incluyente y México abierto al mundo), cada uno con sus líneas de acción (propuestas de campaña) estaba cruzado por tres causas transversales: 1) desarrollo sustentable, 2) igualdad de género sustantiva (este último concepto no es utilizado por ninguno de los partidos de la coalición Juntos Haremos Historia) y 3) apuesta por la juventud. Todas estas causas tenían, también, sus respectivas líneas de acción.

Después de reconocer los avances logrados en nuestro país con respecto a la igualdad entre hombres y mujeres (paridad en las cámaras, igualación de la matrícula escolar en todos sus niveles), el PRI sostiene que la igualdad entre hombres y mujeres es un tema pendiente en el que nuestra sociedad debe trabajar, por lo que deben implantar acciones que reduzcan las brechas y disparidades entre varones y mujeres. Reconoce lo que las feministas han señalado desde muchos años atrás: el desarrollo de una sociedad, en cualquiera de sus niveles, sólo puede lograrse si desaparecen las brechas de género (Serret, 2008). Las acciones propuestas para tal fin fueron:

1. Combatir la violencia de género: desde la educación para prevenirla, hasta la sanción legal de quienes la ejerzan.

2. Reducir y erradicar las brechas salariales con mecanismos legales que obliguen a patrones a pagar igual a hombres y mujeres que realicen las mismas actividades.

3. El empoderamiento económico de las mujeres facilitando su acceso al mundo laboral, particularmente a las madres, promoviendo su capacitación laboral y la conciliación de este espacio con el familiar.

4. Incrementar guarderías.
5. Un modelo educativo que incluya la igualdad de género y eduque desde temprana edad para conseguirla, e

6. Impulsar programas enfocados en la salud de las mujeres, que prevengan los embarazos adolescentes y la mortalidad maternoinfantil.

Como se aprecia, la plataforma electoral del Revolucionario Institucional considera las seis acciones (que incluyen los aspectos económico, cultural-educativo y de salud) como transversales en sus propuestas de gobierno.

Por su parte, el PVEM, a diferencia de los demás partidos, no menciona en su plataforma electoral el género como un eje transversal de la política pública. ${ }^{7}$ Su propuesta está integrada por seis grandes temáticas: 1) seguridad, 2) economía, 3) medio ambiente, 4) salud, 5) social y 6) política. Es en el quinto punto donde se desarrollan una serie de estrategias en las cuales el género es central, aunque no esté así explicitado.

En dicho rubro se proponen políticas públicas concisas. La primera se concentra en modificar la legislación para que la crianza de las y los hijos sea un asunto de corresponsabilidad (una responsabilidad compartida por el padre y la madre). En un segundo momento postulan medidas jurídicas legales para establecer la equidad salarial y erradicar la brecha salarial entre varones y mujeres. En tercer lugar, el PVEM buscaría sancionar a quienes insulten o intimiden a una mujer por amamantar a sus bebés en espacios públicos. En el tema de violencia, como último punto, su propuesta estuvo encaminada a incorporar como materia, en el sistema educativo, el combate a la violencia, dentro de la cual se incluye la violencia de género.

El contenido en materia de género, o sobre las cuestiones de las mujeres, de las plataformas electorales del PRI y el PVEM es el más parecido entre sí, a diferencia de las demás coaliciones. ¿Cómo se tradujo esto en sus actos públicos, a ras de suelo?

José Antonio Meade realizó 159 actos públicos en la mayoría de los estados de la república. Sólo en 18 de ellos se refirió a los temas de género o cuestiones sobre las mujeres propuestos en su plataforma electoral. De manera general, habló sobre la equidad de género; mas puso énfasis en la reducción de la brecha salarial, guarderías de tiempo completo y el empoderamiento económico de las mujeres con el acceso, de palabra, a créditos. En un par de ocasiones aludió a

6 Partido Revolucionario Institucional, Proceso electoral federal, 2018-2024. Plataforma electoral <https://repositorio documental.ine.mx/xmlui/bitstream/handle/123456789/94944/CGor201801-31-ap-20-2-a1.pdf> [6 de noviembre de 2018].

7 Partido Verde Ecologista de México, Plataforma político electoral, 2018-2024 <https: / /repositoriodocumental.ine.mx/xmlui/ bitstream/handle/123456789/94939/CGor201801-31-ap-20-5-a1.pdf> [6 de noviembre de 2018]. 
los feminicidios, proponiendo ante su público terminar con ellos mediante la creación de un código penal único y una agencia de investigación capacitada. Meade no improvisó en su campaña, se limitó a seguir al pie de la letra tanto la plataforma electoral de su partido como la del Verde. En ningún momento hizo mención sobre los otros temas de género: aborto, matrimonio igualitario, derechos de la diversidad sexual. No obstante, a diferencia de AMLO, su agenda de género durante la campaña electoral fue más diversificada, con una propuesta teóricamente sustentada (el hecho de ver la equidad de género como un tema de justicia y de oportunidad para el desarrollo económico es un ejemplo de ello).

\section{Ricardo Anaya}

Anaya debía condensar, en una sola campaña, una agenda que cristalizara posiciones ideológicas algo opuestas. Por un lado, ser fiel a los principios de derecha y medianamente conservadores (pero con una tradición liberal) de su partido, Acción Nacional, y, de manera simultánea, atender a la ideología de dos partidos de centro izquierda, uno de los cuales, el Partido de la Revolución Democrática, ha convertido a la Ciudad de México, a través de sus administraciones desde 1997, en una capital progresista en cuestiones de género: el aborto está permitido hasta la semana 12 de gestación, las personas del mismo sexo pueden contraer matrimonio y el Código Civil local permite el cambio de sexo y nombre para personas transexuales. No hay que olvidar que el PRD postuló como diputada a Marcela Lagarde, intelectual y feminista a la que le debemos la Ley General de Acceso de las Mujeres a una Vida Libre de Violencia (LGAMVLV).

Las plataformas electorales del $\mathrm{PRD}^{8}$ y de Movimiento Ciudadano $(\mathrm{MC})^{9}$ son documentos diseñados con una visión política que tiene en cuenta la realidad del país desde sus particularidades. Lo que se ve reflejado, también, en lo que atañe a las mujeres y el género. Varias cuestiones son importantes de ser señaladas.

Los dos partidos de izquierda tienen más o menos claro que existe una distinción entre género y mujeres. $\mathrm{El}$ PRD reconoce que las mujeres son un sector social que sufre discriminación y padece de modo más profundo los distintos problemas que aquejan a la sociedad mexicana (pobreza, desempleo, bajos salarios, cargas laborales no remuneradas y violencia en sus múltiples expresiones, como la sexual y la violencia feminicida).

El PRD delinea una serie de propuestas que cobija bajo el nombre "México con inclusión social y equidad”. Aquí se especifica que la perspectiva de género (el reconocimiento de que las relaciones entre varones y mujeres son de poder), la diversidad y la paridad deben ser transversales en las políticas, planes de desarrollo y acciones de gobierno, con el objetivo de construir una sociedad que respete y vigile los derechos de las mujeres; también se propone asegurar la paridad, horizontal y verticalmente, en los puestos de decisión política y en los tres niveles de gobierno. Posteriormente, distintos rubros, entre ellos el de justicia penal, plantean el fortalecimiento de la justica civil de las mujeres, además de la aplicación universal de los protocolos para atender la violencia de género. Como se puede apreciar, el PRD no incluye, en su plataforma, acciones que incidan en aspectos identitarios o de roles sociales tradicionales. En ningún lado aparecen temas que aludan a guarderías, horarios flexibles, créditos económicos a mujeres.

Llama la atención el hecho de que en la plataforma del PRD exista, relacionado con el género, un valor político: la libertad. En su línea de acción "México libre y diverso" se hace mención a la garantía de la libertad sobre el desarrollo de la personalidad, es decir, el PRD

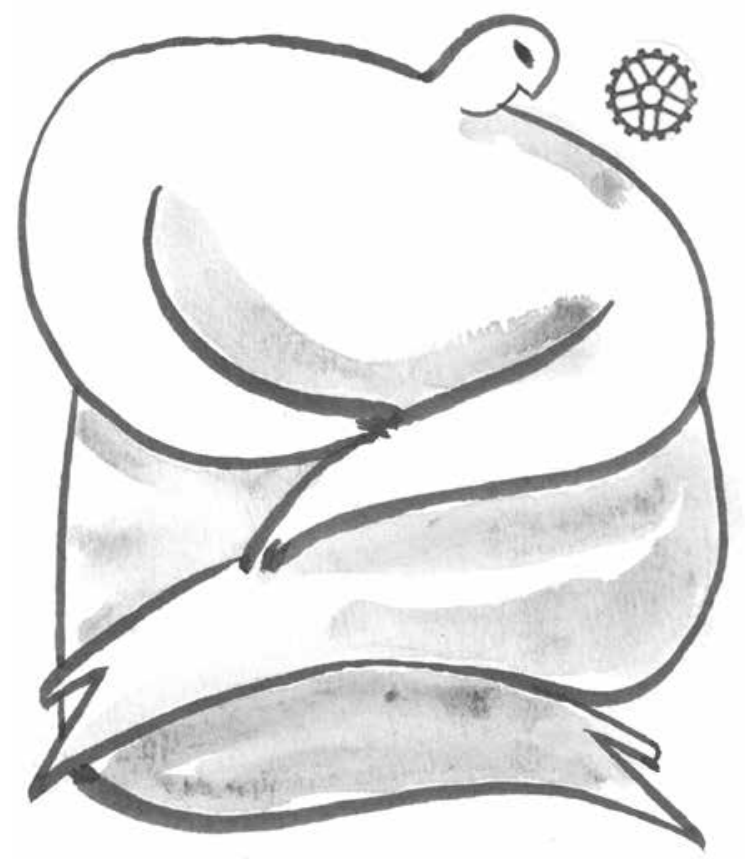

8 Partido de la Revolución Democrática, Plataforma electoral 2018 del Partido de la Revolución Democrática, México sostenible <https: / / repositoriodocumental.ine.mx/xmlui/bitstream/handle/123456789/94966/CGor201801-31-ap-20-3-a1.pdf> [6 de noviembre de 2018].

9 Movimiento Ciudadano, Plataforma electoral, 2018-2024. Un nuevo proyecto de país <https://repositoriodocumental.ine. $\mathrm{mx} / \mathrm{xmlui} /$ bitstream/handle/123456789/94947/CGor201801-31-ap-20-6-a1.pdf> [6 de noviembre de 2018]. 
se comprometió a garantizar la igualdad de derechos de las poblaciones lésbico, gay, bisexual, transexual, transgénero, travesti e intersexual (LGBTTTI) (primera y única vez en todas las plataformas electorales que aparece incluida la agenda LGBT+) y la defensa de todas las formas de cohabitación, el derecho a la identidad y expresiones de género. En teoría, esto se apareja con una de sus líneas políticas más importantes en cuanto a los derechos de las mujeres, el partido del Sol Azteca se comprometió a continuar con el desarrollo de una agenda para que las mujeres puedan decidir sobre su propio cuerpo, es decir, las supone sujetos libres.

En igual tesitura se encuentra MC. En la primera línea de acción de su plataforma electoral contempla el Desarrollo humano para la libertad e igualdad. De nueva cuenta, los dos valores políticos de la modernidad aparecen asociados (Serret, 2016): se plantea la promoción de la igualdad sustantiva de género con 16 acciones aquí sintetizadas: la transversalización de género y el etiquetamiento de recurso específico para ello en los distintos órdenes de gobierno y en las políticas públicas; eliminar todas las formas de violencia y discriminación en contra de las mujeres y niñas, y garantizar el acceso a una vida libre de violencia; promover la participación política de las mujeres y ampliar los espacios de representación política; combatir la feminización de la pobreza, reformas laborales que propicien la creación de espacios de trabajo para mujeres (en particular para madres trabajadoras) y, finalmente, una agenda por la libertad basada en la promoción de leyes que garanticen el derecho de las mujeres a decidir sobre su propio cuerpo. Cabe agregar que, en todas las líneas de acción propuestas por MC, se incluye un punto sobre la equidad de género que atañe de manera particular al tema que se aborda, por ejemplo, en educación, seguridad, desarrollo comunitario, gobernabilidad y empleo.

A lo largo de la plataforma electoral del PAN, ${ }^{10}$ el valor de la libertad está presente, pero en ningún momento se relaciona con la igualdad (o equidad) de género, y este último ni siquiera se localiza en el índice. Sin embargo, la igualdad de género es considerada un elemento que debe ser transversal en las políticas y programas de gobierno.

Entre las principales propuestas de Acción Nacional al respecto, están, entre otras: un gabinete paritario, el acceso e impartición de la justicia con perspectiva de género, aplicar plenamente la LGAMvLV, erradicar los feminicidios, asegurar la igualdad salarial entre hom- bres y mujeres, incentivar la participación económica de las mujeres por medio de becas y créditos, focalizar apoyo para mujeres rurales e instrumentar políticas públicas que garanticen la inclusión social de las mujeres con el principio de la no discriminación.

Durante la campaña, Anaya privilegió un enfoque identitario. A ras de suelo, se limitó a seguir lo delineado por la plataforma electoral de su partido, soslayando las propuestas sustantivas del PRD y MC, lo cual se explica por el origen partidista del candidato. Anaya realizó 159 actos públicos de campaña y sólo en 19 colocó una agenda de género. Los temas que abordó fueron, sobre todo, la brecha salarial, la violencia de género y el feminicidio. Es de destacar que de manera directa fue cuestionado en dos ocasiones sobre el tema del aborto y los matrimonios entre las personas del mismo sexo. En ambas ocasiones dijo estar en contra del aborto y ser tolerante frente a las preferencias sexuales de las personas. El candidato de Por México al Frente contestó a dichas preguntas como si no fuera candidato de dos propuestas totalmente a favor de ambos temas (la despenalización del aborto y el matrimonio igualitario).

Como se puede apreciar, la síntesis de la agenda de género de las campañas presidenciales refleja lo planteado por cada partido contendiente en sus respectivas plataformas electorales, pero tuvieron que tocar temas que surgieron en foros públicos, lo cual es sociológica y politológicamente relevante. Esto se vincula a las demandas de los públicos alternos.

\section{Públicos alternativos: un agente político central en las agendas de género de las campañas presidenciales}

Una agenda político-electoral se construye mediante discusiones en la arena pública. El tema de género (y las cuestiones sobre las mujeres) no es la excepción. Gracias a la emergencia de públicos alternos lo que vimos en las campañas fue una mezcla de:

1. La composición de las agendas por posicionamiento de "temas viejos".

2. Temas emergentes que permiten que las agendas estén en construcción constante.

3. Temas novedosos que atañen de forma directa a las cuestiones de emergencia nacional, esto es, violencia y paridad.

\footnotetext{
${ }^{10}$ Partido Acción Nacional, El cambio inteligente. Plataforma electoral 2018 <https: / repositoriodocumental.ine.mx/xmlui/ bitstream/handle/123456789/94968/CGor201801-31-ap-20-1-al.pdf> [6 de noviembre de 2018].
} 
4. Una paradoja que se expresa en los planos político y cultural, pues lo que se pone en juego son certezas de carácter identitario: se puede sostener que los candidatos no hicieron mención a asuntos de igualdad sustantiva porque éstos no se perciben como electoralmente rentables (matrimonio igualitario, aborto, paridad política), en cambio, sostuvieron, como agenda de género, una serie de cuestiones que incumben al modo en que se concibe lo que es o debe ser una mujer en nuestra sociedad: madre o cuidadora.

A continuación, presentamos algunos posicionamientos de colectivos feministas y de la diversidad sexual.

Grupo de Información de Reproducción Elegida (GIRE). Este colectivo, uno de los más importantes en la lucha por la despenalización del aborto en México, presentó durante las campañas el documento " $51 \%$ una agenda para la igualdad" 11 ante la ausencia de los temas de igualdad en éstas. El documento se refiere al aumento de la violencia contra las mujeres y considera que, aunque hay esfuerzos desde el Estado, instituciones y algunas políticas públicas por atacar el problema, se siguen enfrentando problemas graves de desigualdad de género; esto fue evidente tanto en las posturas de los candidatos a la Presidencia, como en el hecho de que todos fueran hombres. El documento puntualiza que el camino para afrontar la violencia es la transversalización de la perspectiva de género frente a las políticas rosas (propuestas que prescriben cómo debe ser una mujer) y debe haber un ambiente de tolerancia cero ante el acoso y los feminicidios. El documento postula un conjunto de acciones: 1) despenalizar el aborto (medida aprobada únicamente en la CDMx); 2) acceso a la justicia; 3) seguridad social, conciliación y derechos laborales (ratificación del Convenio 189 de la Organización Internacional del Trabajo); 4) democracia paritaria; 5) derechos laborales de trabajadoras del hogar; 6) estrategias para disminuir el embarazo adolescente.

Las defensoras de derechos humanos Feministas Exigimos ${ }^{12}$ pidieron a los candidatos tomar posición frente a 11 temas: Estado laico, acceso seguro y gratuito al aborto, inclusión social, derecho a una vida libre de violencia, derecho universal al cuidado, acceso a beneficios culturales, representación paritaria en la política, entre otros.

El colectivo Las Constituyentes cDMx difundió la Agenda política feminista $2018 .{ }^{13}$ Este grupo se organizó para pugnar por una constitución en la CDMx con perspectiva de género y para el reconocimiento de los derechos sustantivos de las mujeres. La agenda es amplia: 1) Acceso a una vida libre de violencia: alerta de género, investigación de feminicidios, una vida libre de prácticas de trata, penalización al consumo y explotación de los cuerpos de las personas, especialmente de las mujeres y niñas, entre otras. 2) Paridad sustantiva: igualdad de oportunidades en todos los ámbitos; presupuestos etiquetados, tipificación de la violencia política, no a la criminalización social y política de las mujeres. 3) Derechos económicos, sociales y ambientales. 4) Derecho al tiempo y al cuidado: universalidad del cuidado. 5) Disidencias sexogenéricas: eliminar la objeción de conciencia; áreas especializadas para la atención de la comunidad LвтTті. 6) Garantía del Estado laico: libertad de conciencia y creencia, elevar a rango constitucional el derecho de las mujeres al aborto seguro, gratuito, decidir sobre su propio cuerpo, diversidad de familias.

La Coalición Mexicana LGBTtтr,${ }^{14}$ formada por múltiples colectivos como Familias Diversas, acusó que los temas de la comunidad estuvieron ausentes del debate entre candidatos, por ejemplo, el matrimonio igualitario (con excepción del PRD). El colectivo denunció, entre otros, que el matrimonio igualitario no se ha legislado en todos los estados. Sólo en 12 de 32 entidades se pueden casar sin la necesidad de interponer un amparo. Por ello, la coalición demanda la modificación de los códigos civiles. También colocaron otros temas centrales como el de la exclusión y los prejuicios en torno a las familias diversas en las escuelas.

Criticaron a AMLO pues, a pesar de provenir de un partido que cuenta con una Secretaría de la Diversidad Sexual, él no está comprometido con esta agenda. Cabe recordar que AMLO bloqueó la Ley de Sociedades de Convivencia en la Ciudad de México y su posición es que esos temas controvertidos se someterán a consulta. Cuestionaron también la falta de compromiso de Anaya y Meade. La coalición elaboró un documento para

${ }^{11}$ Disponible en: <https: / /gire.org.mx/wp-content/uploads / 2018/05/51-Una-agenda-para-la-igualdad.pdf> [8 de noviembre de 2018].

12 http://ejesur.com.mx/2018/06/04/elecciones-mexico-2018-feministas-exigimos-compromisoporlaigualdad/ [8 de noviembre de 2018].

${ }^{13}$ El documento está disponible en: <https: / / lasconstituyentescdmx.files.wordpress.com/2018/06/agenda_feministacdmx20182.pdf> [6 de octubre de 2018].

${ }^{14}$ https://www.animalpolitico.com/2018/03/inclusion-laboral-erradicacion-violencia-lgbt-candidatos/ [8 de noviembre de 2018]. 
todas/os las/os candidatas/os, en todos los niveles, cuyo eje era erradicar la discriminación y visibilizar una agenda de derechos humanos, salud integral sin discriminación, educación para la inclusión y no discriminación, derechos laborales e inclusión económica, cultura incluyente y medios de comunicación, participación política de la comunidad y Estado laico.

Así, frente a las campañas y el tratamiento tradicional de temas "de mujeres" y la comunidad Lвттті, estos públicos presentaron agendas con reivindicaciones libertarias y de derechos sobre el cuerpo y formas de vida alternas. En síntesis, este grupo se propone visibilizar problemas de la comunidad tanto en materia de acceso a servicios de salud como de creación de una ciudadanía sexual que normalice la diversidad sexual con un enfoque de derechos humanos (López, 2017).

\section{Reflexiones finales}

Tras lo expuesto, es posible apreciar tendencias contradictorias en el escenario político nacional. A continuación las enumeramos.

1. La paridad ha permitido avances fundamentales en materia de política pública y en cuanto a número de mujeres en el Congreso. En la actualidad tenemos $48 \%$ de mujeres en la Cámara Baja y $49 \%$ en el Senado; congresos locales paritarios, aunque solamente hay tres gobernadoras $y$ $11.9 \%$ de alcaldesas. La paridad, sin duda, debe continuar siendo un tema central en la agenda, sobre todo para conseguir mayor presencia de las mujeres en estos niveles de representación política.

2. A pesar de que la democracia paritaria es un principio constitucional desde 2014 , las campañas fueron ambivalentes o tímidas, por decir lo menos, en relación con el principio de igualdad de género.

3. Junto a ese conservadurismo, presenciamos que varios colectivos colocaron temas progresistas de reivindicación de derechos y libertades.

El supuesto de la teoría de la masa crítica, según el cual a más mujeres, más agendas y políticas de género, es cuestionable, como se ha demostrado con estudios de otros países (Childs y Krook, 2006). Los temas relativos al género fueron muy marginales en las campañas presidenciales de 2018. Lo que predominó, como en las campañas presidenciales de 2012 (Cerva y Cornejo, 2014), fueron acciones y propuestas que forman parte de las políticas redistributivas y de reconocimiento. Es decir, por un lado, una serie de lineamientos políticos, particularmente en la esfera de lo económico (o de los derechos materiales), que intentan paliar, estrechar y, en el mejor de los casos, desaparecer las inequidades, desigualdades e injusticias heredadas del liberalismo (económico), y, por otro, un conjunto de propuestas que prescriben cómo deben ser las mujeres en nuestro país o los espacios que deben ocupar, tales como madres, esposas, criadoras y encargadas del hogar.

Queremos insistir en que tanto en 2012 como en 2018 hay algunos temas de género presentes en las campañas; pero, más que políticas por la igualdad de género, son políticas para mujeres. La primera remitiría a un conjunto de planteamientos (políticos y éticos) que promuevan el pleno ejercicio de los derechos políticos y sociales no sólo de las mujeres, sino también de las personas con una identidad de género diferente de la hegemónica, sin que primen sus aspectos adjetivos. La segunda comprende únicamente acciones que están focalizadas a un grupo identitario particular, en efecto, políticas distributivas y de reconocimiento.

De alguna manera ambos enfoques estuvieron incluidos en las propuestas políticas de los contendientes; no obstante, aunque todos los partidos consideran que la equidad y la perspectiva de género deben ser principios rectores y transversales del ejercicio del poder político, tal y como se expone en sus plataformas electorales (algo que, estaríamos de acuerdo, se puede colocar dentro de la política de la igualdad), todas las fuerzas políticas terminaron por proponer, sobre todo a ras de suelo, acciones y políticas públicas que remiten a las mujeres como pertenecientes a un grupo de personas con necesidades particulares y ello no se debe, subrayamos, a una miopía conceptual del término género, sino a un buen entendimiento de lo que significa reconocer todas las característica adjetivas y llevarlo al terreno de lo político.

El ejemplo más claro de ello es la postura del PEs. En su afán por proteger la vida desde la concepción y la forma heterosexual de la familia, hace patente una de las principales imágenes sociales de las mujeres, como esposas, amas de casa y criadoras de las y los hijos. De ahí que todas sus propuestas estén encaminadas a beneficiar a las mujeres en cuanto esos roles.

El tricolor comprende la equidad de género como un ámbito que se reduce de modo exclusivo a una brecha salarial, empoderamiento económico de las mujeres y a la posibilidad de hacer compatible el mundo laboral con el familiar. Incluso uno de sus eslóganes de campaña fue el famoso y criticado por las feministas: "salario rosa". Se trata de una política pública de 
desarrollo social que opera en el Estado de México y cuyo principal objetivo es paliar la pobreza extrema; y sólo pueden acceder a dicho recurso las mujeres que se dediquen a las labores del hogar. Se trata de una política redistributiva, pero que al mismo tiempo reconoce la particularidad del individuo al que está dirigida.

Los postulados en campaña de la coalición Por México al Frente, desde su facción panista, se concentraron en el empoderamiento económico, en la maternidad, en la conciliación entre el horario laboral con el familiar y las estancias infantiles. Otra vez, todo centrado en un reconocimiento de las mujeres con necesidades particulares en cuanto madres de familia que, sin embargo, laboran.

¿Todas estas propuestas están determinadas por una visión que conjuga las políticas distributivas y del reconocimiento? Las políticas públicas que se pueden derivar de cada una de esas propuestas se focalizan en un grupo de individuos que responden a características socialmente imputadas cuyo fin es la erradicación o, en el mejor de los casos, la reducción de las desigualdades económicas, pero no potenciar el pleno ejercicio de la ciudadanía de esos individuos, en este caso, las mujeres y, claro está, de aquellas personas cuyas identidades genéricas no corresponden al modelo hegemónico.

Recordemos que en sus plataformas electorales algunas de las izquierdas mexicanas (PRD y MC) diseñaron una propuesta de carácter ético-político sobre la igualdad de género, que reconoció de manera fundamental la dimensión de la libertad de los individuos, haciendo énfasis, por supuesto, en las mujeres y en las personas de la diversidad sexual. Los planteamientos de dichos partidos no son políticas para las mujeres (o para las personas de la diversidad sexual), son políticas por la igualdad en la medida en que se contempla, como mandato, la libertad de los individuos que componen el orden social. Ahora bien, si ese planteamiento estaba en sus plataformas electorales, ¿por qué no fue llevado a las campañas a ras de suelo? No se trata de una miopía; o, en todo caso, ello responde a cuestiones de carácter identitario y, por lo tanto, de seguridad ontológica.

No queremos decir que no deban existir propuestas políticas que atiendan el reconocimiento de las personas pertenecientes a un grupo. Una agenda feminista partiría de un enfoque en el cual toda la agenda política esté transversalizada por una perspectiva de género, lo que quiere decir que en cada uno de los rubros (económico, social, cultural, salud, educación, seguridad) se deben hacer visibles las relaciones de poder entre lo masculino y lo femenino.

\section{Fuentes}

BarRagán, Almudena

Y DARINKA RODRÍGUEZ

2018 "Cinco feministas opinan sobre 'Femsplaining', las propuestas de género de López Obrador", en El País, $1^{\text {o }}$ de junio <https: / / verne. elpais.com/verne / 2018/06/02/mexico / 1527892253_983452.html> [8 de noviembre de 2018].

Cerva, Daniela y Eduardo Cornejo

2014 "Entre la ausencia y lo insulso: la agenda de género en las campañas presidenciales de 2012", en Ação Midiática. Estudos em Comunicação, Sociedade e Cultura, núm. 7, pp. 1-24.

Childs, Sarah y Mona Lena KroOK

2006 "The Substantive Representation of Women: Rethinking the 'Critical Mass Theory', ponencia presentada en la Annual Meeting of American Political Science Association, Washington, D. C.

Coвo, Rosa

2004 "Sexo, democracia y poder político", en Feminismos, núm. 3, pp. 17-29.

Fraser, NANCY

1993 "Repensar el ámbito público: una contribución a la crítica de la democracia realmente existente", en Debate Feminista, vol. 7, pp. 23-58.

Kanter, Rosabeth Moss

1977 "Some Effects of Proportions on Group Life", en American Journal of Sociology, vol. 82, núm. 5, pp. 965-990.

López, JAIRo ANTONIO

2017 "Los derechos LGBT en México: acción colectiva a nivel subnacional", en European Review of Latin American and Caribeean Studies, núm. 104, pp. 69-88.

Reyes-Housholder, Catherine

2018 "Presidentas, poder y cambios pro-mujer en Chile y Brasil", en Lucía Miranda Leibe y Julieta Suárez-Cao (eds.), La política siempre ha sido cosa de mujeres: elecciones y protagonistas en Chile y la región, Facultad Latinoamericana de Ciencias Sociales, Santiago de Chile, pp. 25-40.

SAPIRO, VIRGINIA

y Pamela Johnston Conover

1997 "The Variable Gender Basis of Electoral Politics: Gender and Context in the 1992 US Election", en British Journal of Political Science, vol. 27, núm. 4, pp. 497-523.

SCHAFFNER, BRIAN

2005 "Priming Gender: Campaigning on Women's Issues in U.S. Senate Elections", en American Journal of Political Science, vol. 49, núm. 4, pp. 803-817.

Serret, Estela

2008 ¿Qué es y para qué es la perspectiva de género?, Instituto Oaxaqueño de la Mujer, Oaxaca de Juárez.

Serret, Estela

2016 "Igualdad y diferencia: la falsa dicotomía de la teoría y política feministas", en Debate Feminista, núm. 52, pp. 18-33.

Studlar, Donley T. E IAN McAllister

2002 "Does a Critical Mass Exist? A Comparative Analysis of Women's Legislative Representation since 1950", en European Journal of Political Research, vol. 41, núm. 2, pp. 233-253. 
Anexo. Cuadro comparativo de las agendas de género en las plataformas electorales y las propuestas de campaña de los partidos contendientes

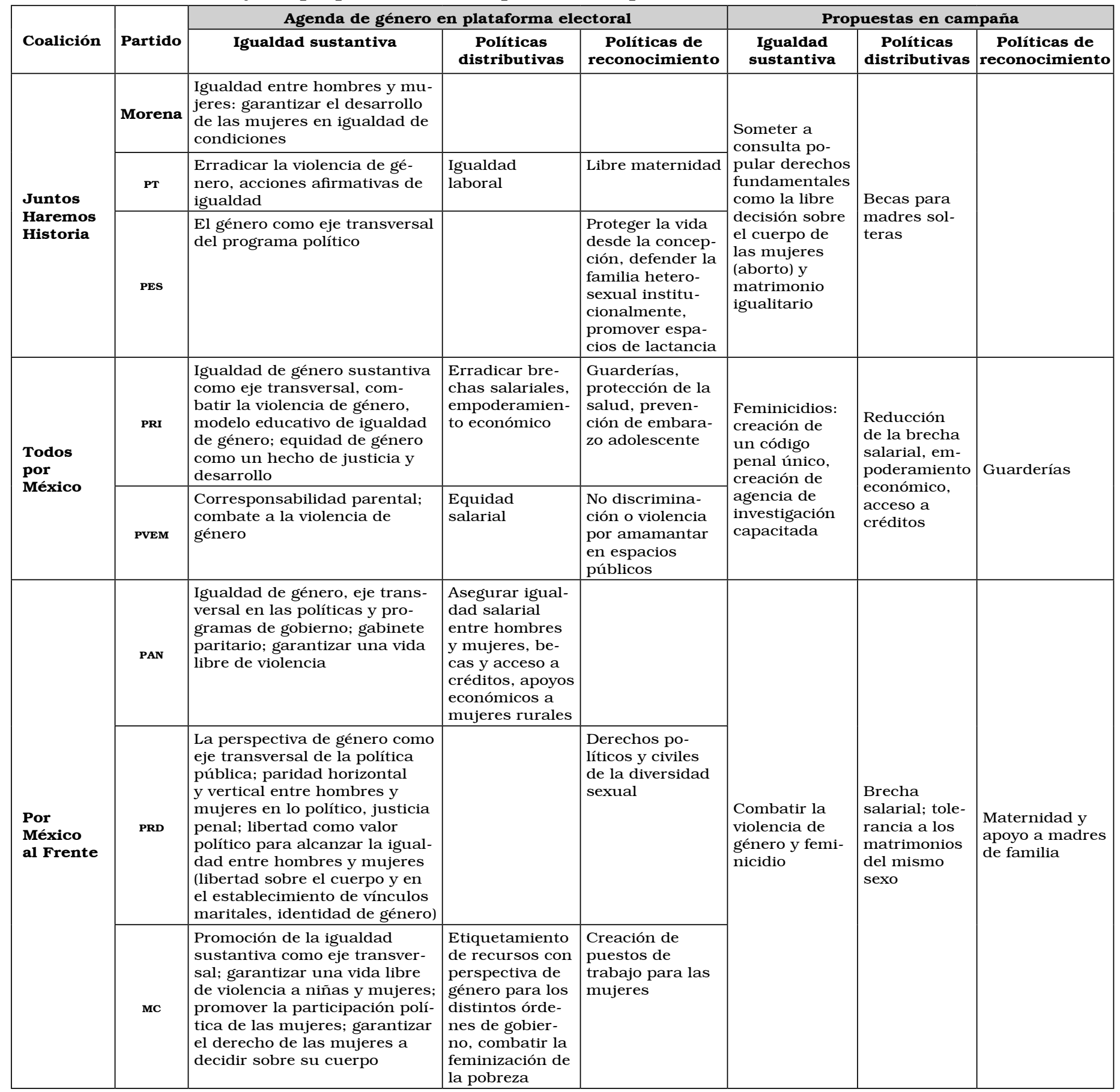

Fuente: Elaboración propia, con datos de las plataformas electorales de los partidos políticos contendientes y de la base de datos de los autores.

Se puede apreciar que, a pesar de que todos los partidos (con excepción del Verde Ecologista) plantearon en sus plataformas electorales al género como eje transversal de la política pública y la igualdad de género como un objetivo importante, ello no se tradujo en posicionamientos de campañas. Dichos planteamientos se vieron plasmados en propuestas de campaña con enfoque de políticas distributivas y de reconocimiento. No obstante, por el contexto nacional actual, el combate a la violencia de género y los feminicidios fueron temas centrales para Anaya y Meade, lo cual puede ser localizado dentro del rubro de la igualdad sustantiva. Finalmente, no deja de ser interesante -y por ello hay que resaltarla- la postura de AMLo sobre llevar a consulta popular temas como el aborto y el matrimonio igualitario, lo que va en contra de la igualdad sustantiva, porque los derechos no pueden ser sometidos a consulta popular. 